\title{
Approach to the Study of Kinematics and Modeling Grip of 22 DOF Anthropomorphic Gripping Manipulator
}

\author{
Ivan V. Krechetov* and Arkady A. Skvortsov \\ Office of Scientific Research and Development, Moscow State University of Mechanical \\ Engineering (MAMI), Moscow, Russian Federation; krechetov_ivan@mail.ru
}

\begin{abstract}
The results of analysis and study the kinematic model of the anthropomorphic arm with 22 degrees of freedom are given. Describes how to use the package GraspIt! virtual simulation and experimental study of the grasping efficiency of various items. Applications submitted technique enables to compare different versions of kinematic schemes of manipulators and to determine the adequacy of the choice of a particular kinematic scheme for the optimal grasp of a known set of objects. This approach is relevant in the development of manipulators grip, especially in the presence of restrictions on the number of controlled degrees of freedom. For example, when developing bionic prostheses hands actual task of reducing weight and, correspondingly, the number of degrees of freedom. The paper compared the two kinematic schemes to capture the geometric primitives example, so-called human scheme - when the thumb is opposed to the little finger, and monkey scheme - when the thumb is opposed to the middle finger. It is shown that to perform the grasp of large objects appropriate to use human scheme.
\end{abstract}

Keywords: Anthropomorphic Grip, GraspIt, Manipulator Kinematics, Object Grasping, RUbionic, Virtual Modelling Manipulator

\section{Introduction}

\subsection{Introduce the Problem}

Contemporary society faces the challenge more and more coming from the growth of the costs of menial work.

Now we live in the sixth technological and economic paradigm and the leading hand in it is given to the development and evolution of service and personal robot technique. Rational robots are little by little penetrating into all spheres of activities and experiences that constitute a person's normal existence - from toys to assistants for nurses and waiters who execute family responsibilities in the care of disabled people. Also, these robots can execute menial work, for example, shipment and unloading conveyor belts, sorting products, prepacking and packing competitive products.
The necessity inside firms for intelligent control systems and on-line manufacturing system worldwide manipulators becomes at a continually increasing rate actual with every year. By expert opinions ${ }^{1}$ next years, a lot of assembling technological lines will be supplied with manipulators. For raising the execution of technological lines is the applying of resembling a human being type general grippers, able to adapt the difficult geometrically formed items, which have the capability to advance and uphold the grip, capable to modify the contour of the grip.

Under usual conditions when we use special operators with certain similarity with the kinematics of the human hand, for example the Barrett Hand ${ }^{2}$, involving grippers. Nevertheless, there is a great number of humanlike manipulators, for example, the DLR II, Meka Hand ${ }^{3}$, the Shadow Dexterous Hand ${ }^{4}$. The present objective is

\footnotetext{
${ }^{*}$ Author for correspondence
} 
the optimization of the usual pattern of the manipulator to reach an equilibrity with the levels of liberty and the capability to accommodate the manipulator to a various item forms.

There is a great number of activity and a huge growth of fundamental research activities and developments and into the sphere of grip manipulators and anthropomorphic robots. The human hand symbolizes a general biomechanical instrument which lets the execution of mechanized operations and also to grip items of accidental form reliably through applying of the fingers. And so, the rules of bionics are becoming widespread more and more among makers of systems and technical equipments ${ }^{5}$.

In the progress of resembling a human being pickups the next contrasting trends are frequently used:

A growth in the number of regardless controlled levels of liberty in the hand. Gripping devices with many levels of liberty have bigger elasticity, agility, which we can use for execute more difficult objectives, which demand the exact manipulation of items with difficult form. This is particularly normal for remotely operated anthropomorphic robots, which operate in a mode which imitates the motions of the operator.

A lowering in the number of regardless levels of liberty. A considerable number of regardless levels of liberty and feedback transmitters meaningly make difficult the manipulator monitoring system.

Applying kinematically related transmission of motion from median to distal phalanx the force of the grip is greatly restricted, mainly with items of little geometric measurements, in that with replete flection of the finger the distal phalanx is in succession quite turned adjoining the phalanx proximalis. In this way, the principle effective areas employed when gripping space-saving items are the proximal and the distal phalanx, meanwhile the median phalanx is principally serving to supply with a bigger degree of mobility to the distal phalanx and produce flexibility to the area of the item.

Manipulators with kinematically related swivel nodes have a restricted capability to accommodate to the form of an item. But in spite of that, the diversity in the gripping effectiveness can become a scanty argument for their utilization in autonomous anthropomorphic manipulator designs for the reason that supplementary operated levels of liberty demand more actuators that are not at all times well-founded.

The earliest research is a judgment of the efficacy of the kinematic scheme of a manipulator made for gripping items. The objective of this study is to examine the points of view to the modeling procedure coming from the gripping of items from a predetermined set and of course the research of the degree of hold upon an item shown by the manipulator founded on the metric suggested ${ }^{6}$ in the movement of a recently advanced resembling a human being handling manipulator.

An anthropomorphic hand has distinctions compared with the hand of monkeys. As for people, the plane of the thumb is opposing to the little finger, which lets the fingers to be turned. As for monkeys, the thumb is opposing to the middle finger. These two schemes are the purposes of this research.

It is essential to manage the position of the executive gripping body when there are grip functions on items with difficult form. The purpose of control of many finger grips comes to this control where the executive body of every finger moves on its special trajectory. The trajectory of movement shows the orientation in space.

\subsection{Investigate the Importance of the Problem}

There are models of anthropomorphic robots with arms and multiple-link grippers able to execute difficult movement of items inside the environment. In the world market a significant range of various robotic handling devices is shown. The most nearly belonging to the presented in this work in terms of design and technology are: Anthropomorphic Hand SAH (SCHUNK), Shadow Hand (SHADOW ROBOT COMPANY LTD), the DLR Hand II (German Aerospace Center).

These manipulators let the adaptation and application of developed robotic systems in an environment, which provide the capability to grip, manipulate hold and items of geometric form. The principle disadvantage of the manipulators is restricted availability caused by their main purpose.

As a means to guarantee that personal and industrial anthropomorphic manipulator robotics technology is generally available. Applying the experience of making a gripping manipulator ${ }^{3,4,7,8}$, the points of view to mathematical simulation ${ }^{9-11}$ and control of the procedure of handling items ${ }^{12}$, we are doing research whose main purpose is the evolution of a kinematic scheme for a manipulator to grip items which are represented by the easy geometric forms: Sphere, cube, cylinder. The consequences of this study will be used for developing a great number of final products: 
- Bionic prosthetic hand.

- General anthropomorphic gripping device with accommodations to execute gripping tasks for activity in robotic systems and as robotic assistants

\subsection{Related Work}

The main and important approaches in the evolution of procedures for monitoring used when gripping items are:

- An adaptive synthesis of directions to manage the grip using tactile sensory information.

- The summarizing of an immutable set of movement patterns for gripping a restricted class of items with a recognized or defined form using contractive force.

- Execute motions which imitate an operator's activity managed by information received from a gripping device.

The given method in $^{13}$ of the synchronous motion control of a multijointed manipulator can be enclosed to the management of the grip of items with various forms providing different types of handling. There are two points of view to the method of grip control. The first point of view is founded on determinant an ensemble of points on the area of the gripped item in this way that their positional relationship, as against the mass center at the application of applied forces, provides static balance, or as it is called the geometric grip. In this situation the gripping of an item and the computation of trajectories of motions of the fingers apply the inverse kinematics method. The point of view, which is founded on human experience, is experiential and founded on the classification of items. The object grasping process is divided into different phases: a tentative grasp, straight grasp of the item and next stabilization of the item. The present strategy ${ }^{13}$ of aligning for the movement of distinct fingers is founded on received information from a system of tactile sensors and its research during the grasping phase. Making contact with the item the direction of vectors of advanced force at the moment of contact are defined and the trajectory of the links are presented improvements which may decrease the magnitude of the tangential component of force which in succession grows the effort of compression on the item and grows the energy efficiency of the actuator's actuating mechanism.

Design of the industrial control for gripping items with various forms, volume and also structure is a difficult problem for the reason that the parameters of motion and necessary continuing efforts of personal links can't be promptly defined. The mathematical statement of the problem of gripping an item by the multijointed manipulator is decreased to solving systems of simple coupling the space of contact points on the area of the item.

As a means to decrease the computational complexity of the problem, most designs are calculated for working with a concrete set of template items ${ }^{14}$. The position of the item as against the manipulator, its orientation, weight, shape are defined for the patterns.

The statement of the movement trajectories of the links connected to a special model of movement can be performed by defining the primary and definitive position in the space. The angular values for the rotary nodes may be determined by resolving the inverse kinematic issue. In works ${ }^{15,16}$ a method to decrease the factor of difficulty dependent on the objective of projection grip functions for various kinematic schemes of manipulators by using eigengrasp was demonstrated. The management problem can be accumulated and given by way of combinations of the 3 groups of eingengrasp:

- Motion of the fingers aside (sideward movement).

- Pressure of the fingers into a closed hand position.

- Motion of the thumb aside.

To the next 3 phases it can be decreased:

- Define the trajectory of the hand to install the handling manipulator in the right position around the item.

- Define the trajectory when the fingers to come into contact with the right orientation and the item in the right place.

- Apply the optimal effort, on the item, that is necessary for a sustained hold.

- In reality, investigators are confronted with the necessity to develop a great number of prototypes for various items. The literature on the biomechanics of the human hand, seventeen singular classes of patterns, which a human uses during management environmental objects meeting in daily life has been cataloged.

Industrial control founded on the models of movements can be advanced by a system of tactile sensory communication for more exact coordination of the motion of fingers during contact with the item. These systems of administration don't demand highperformance computing. Using movement models for gripping items, the most necessary phase is the choose of the most convenient model. Using a 3-finger, 5-finger grip is usually used by people in their life. Formats with 2, 4 fingers are not often used. 
The method of control of a resembling a human being manipulator's fingers and review of the telemetry data from a sample task ${ }^{4}$ has been studied. The telemetry data archive of the rotational positions of the articulations in the hand of the operator using virtual reality gloves was accumulated. In all experiments the calendar progress chart representing the trajectory of the motions of the various fingers corresponds in frequency and distinguishes in amplitude, phase.

The defined models of gripping lets the holding of nondeformable items of elementary geometric forms:

- An apartment object (a paper, plate).

- Cube/box.

- Slender cylindrical item (fork, pencil, pen, spoon).

- Thick cylindrical item (hammer, pipe).

Autors in ${ }^{15}$ showed that a spatial model of an accidental item being gripped can be given as diagram, the main components of which are easy geometric primitives, for example, a box, a sphere, a cylinder.

In another works $^{9-11}$ showed approaches which emphasize the mathematical simulation of dynamics and kinematics. The probability of integrating known patterns from CAD solid modeling lets the elasticity to customize the characteristics of magmatic patterns showing the kinematics of the motion, make modifications to the maintenance guide. The principle disadvantage of MATLAB is that there is no a modeling environment for contact interaction between three-dimensional models. MATLAB provides spacious opportunities for item identification and also modeling nonlinear control systems.

\section{Kinematic Scheme of the Manipulator}

During the research and study of the bodily machinery of the human hand the authors advanced a scheme for a many-fingered hand. The manipulator is comprising five finger sections, every section has four levels of liberty.

In Figure 1 the thumb rotational angle (F5) is 45 degrees (human scheme). In the study a thumb rotational angle of 22.5 degrees was used too (monkey scheme).

\subsection{Explanation of the Knematics of a Finger} We examine the straight kinematic objective for a finger, which consists of three phalanges, simultaneously that we examine the movement in all four joints. We have pointed the rotational angles in the joints as $q_{0}, q_{1}, q_{2}$, and $q_{3}$ thereafter (Figure 2).

(Type 1)

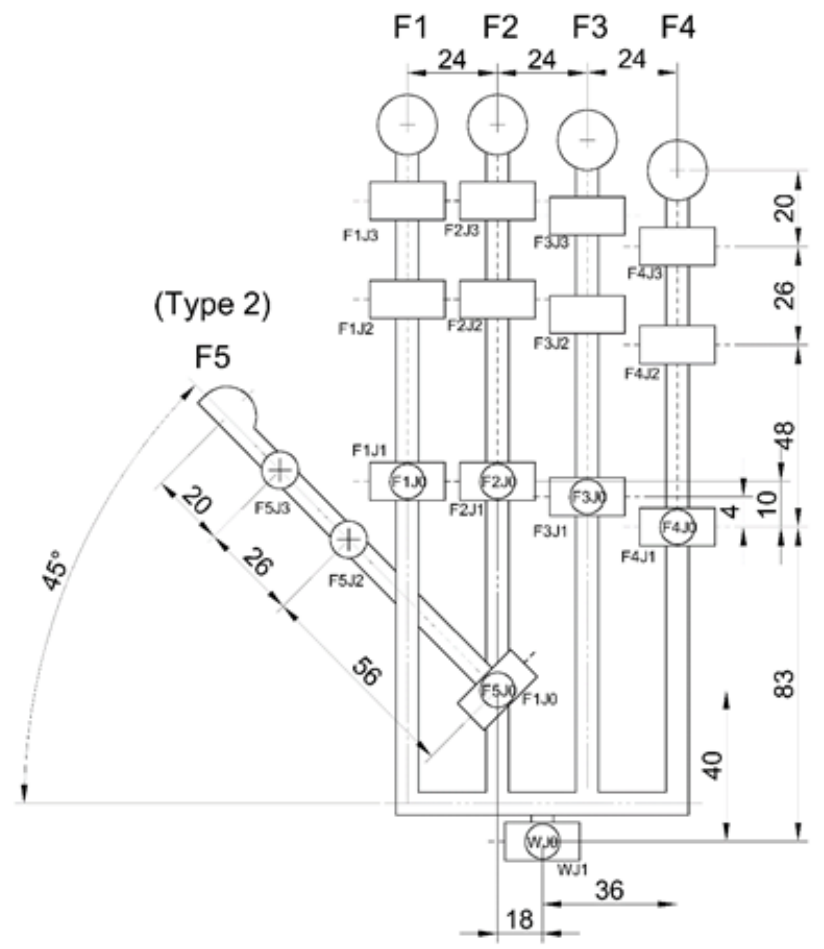

Figure 1. Kinematic scheme of the manipulator.

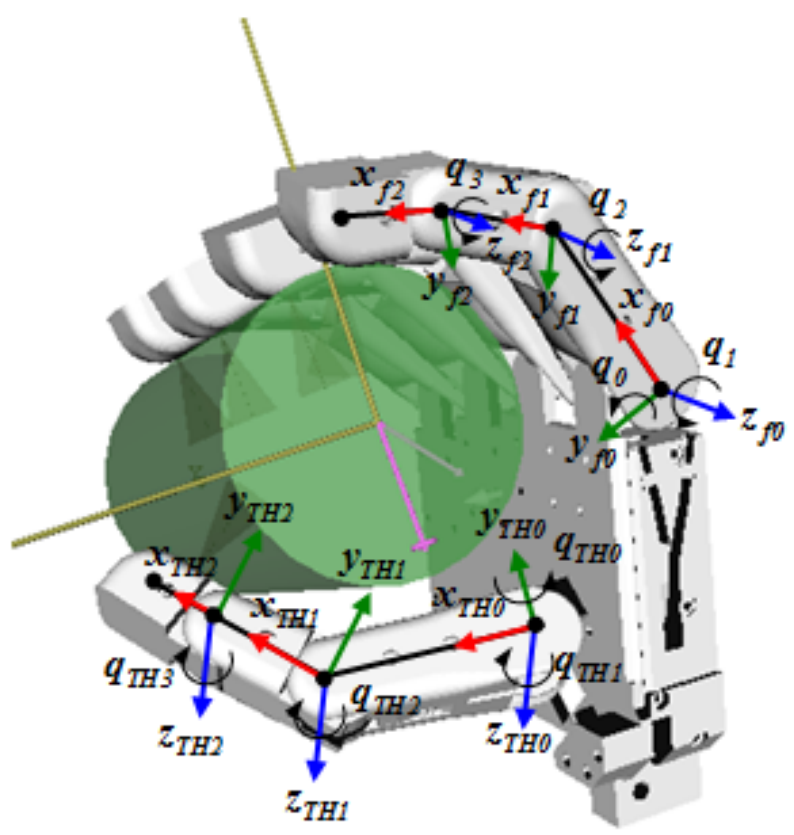

Figure 2. Scheme of the finger depicting the used coordinate systems and angles. 
Also it is better to mention the following: the ranges of permitted values for $q_{0}, q_{1}, q_{2}$, and $q_{3}$ must be narrowly defined. It is necessary to comply with the condition $q_{1} \geq$ $0, i \in\{1,2,3\}$ We have defined the lengths of the phalanges as $l_{1}, l_{2}$, and $l_{3}$. Every joint is related with the frame of reference thanks to a proper index. Fixed coordinate systems are indicated as $O_{f} x_{f} y_{f} z_{f}$ In figure 2 the point of origin $O_{f}$ is related with the earliest or the first articulation. The hand - in the plane $O_{f} x_{f} z_{f}$.

The coordinate transformation matrix $A_{i}^{j}$ is written from $i$-th coordinate system in the $j$-th. In the axonometric homogeneous, the coordinate assignment is a $4 \times 4$ matix.

$$
\begin{aligned}
A_{f}^{0} & =\left(\begin{array}{cccc}
\cos q_{0} & 0 & -\sin q_{0} & 0 \\
\sin q_{0} & 0 & \cos q_{0} & 0 \\
0 & -1 & 0 & 0 \\
0 & 0 & 0 & 1
\end{array}\right) \\
A_{i-1}^{i} & =\left(\begin{array}{cccc}
\cos q_{i} & -\sin q_{i} & 0 & l_{i} \cos q_{i} \\
\sin q_{i} & \cos q_{i} & 0 & l_{i} \sin q_{i} \\
0 & 0 & 1 & 0 \\
0 & 0 & 0 & 1
\end{array}\right), i \in\{1,2,3\}
\end{aligned}
$$

At that,

$$
A_{i-1}^{i} A_{i}^{i+1}=A_{i-1}^{i+1}
$$

Then it can be written that,

$$
A_{f}^{3}=A_{f}^{0} A_{0}^{1} A_{1}^{2} A_{2}^{3}
$$

for example,

$A_{f}^{3}=\left(\begin{array}{cccc}\cos q_{0} \cos \left(q_{1}+q_{2}+q_{3}\right) & -\cos q_{0} \sin \left(q_{1}+q_{2}+q_{3}\right) & \sin q_{0} & \cos q_{0}\left(l_{1} \cos q_{1}+l_{2} \cos \left(q_{1}+q_{2}\right)+l_{3} \cos \left(q_{1}+q_{2}+q_{3}\right)\right) \\ \sin q_{0} \cos \left(q_{1}+q_{2}+q_{3}\right) & -\sin q_{0} \sin \left(q_{1}+q_{2}+q_{3}\right) & -\cos q_{0} & \sin q_{0}\left(l_{1} \cos q_{1}+l_{2} \cos \left(q_{1}+q_{2}\right)+l_{3} \cos \left(q_{1}+q_{2}+q_{3}\right)\right) \\ \sin \left(q_{1}+q_{2}+q_{3}\right) & \cos \left(q_{1}+q_{2}+q_{3}\right) & 0 & -l_{1} \sin q_{1}-l_{2} \sin \left(q_{1}+q_{2}\right)-l_{3} \sin \left(q_{1}+q_{2}+q_{3}\right) \\ 0 & 0 & 0 & 1\end{array}\right)$

Using these matrices, we can use presented angles $q_{0}$, $q_{1}, q_{2}, q_{3}$ and the length of phalanges $l_{1}, l_{2}, l_{3}$, to identify the coordinates $(\mathrm{x}, \mathrm{y}, \mathrm{z})$ of the substance of the manipulator.

There are two different types of finger:

- "The index" (Type 1) -for fingers 2, 3, 4, 5.

- “The thumb" (Type 2) - for finger 1 .

\section{Method}

\subsection{Simulation of the Robot Kinematics}

Based on the kinematic scheme, applying the Solidworks package, the solids modeling of the manipulator has been advanced (Figure 3). The motion parameters were checked and studied, to guarantee a liberty of action like the liberty of motion of a human hand.

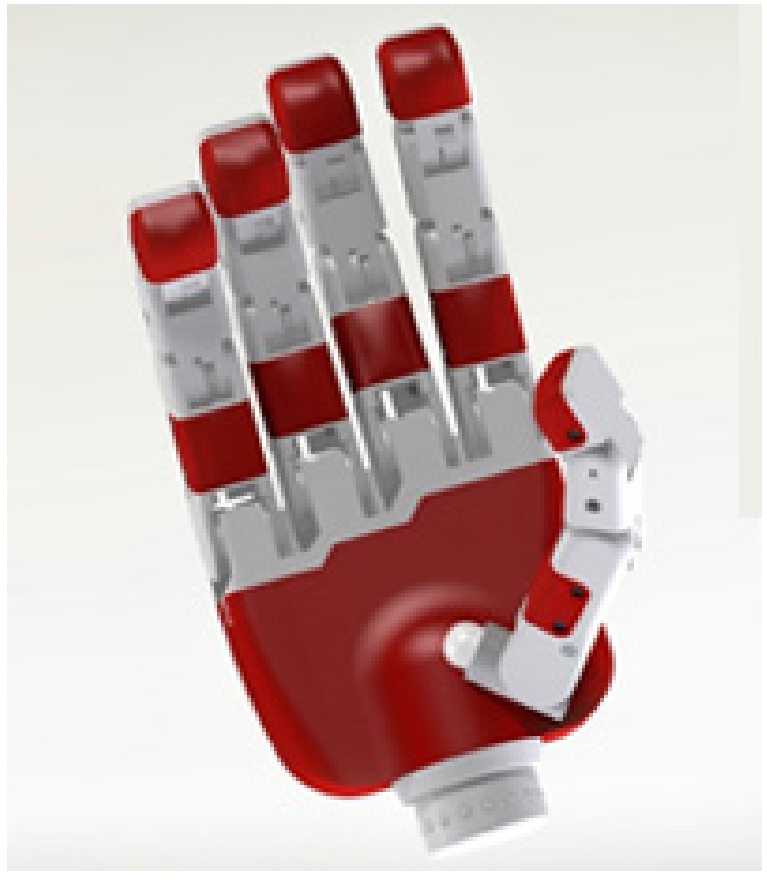

Figure 3. The three-dimensional model of the manipulator in solidworks (CAD).

A pattern of the manipulator was particularly ready for work inside the GraspIt! package which was used to help to the study of the efficacy of item holding by various manipulators. GraspIt! provides the probability of demonstrating the kinematically subordinate links, when the motion of joints is made according to the motion of the guide link.

The organization of complication is defined by the number of spider bevels in the model and appreciated as $o(N)$,

Where,

$N=n_{1} \cdot n_{2} \cdot n_{3} \cdot n_{4}$

$n_{1}$ - The number of spider bevels in the model of the manipulator.

$n_{2}$ - The number of spider bevels in the model of the item.

$n_{3}-$ Number of directions for each couple of spider bevels subject to the full contact interaction.

$n_{4}$ - Quantity of accidental displacements for the couples of manipulator spider bevels to the spider bevels of the item.

Examine a situation with a comparatively little number of repetitions, if $n_{2}=100, n_{3}=24 n_{4}=10$. The 
three-dimensional model of the manipulator includes 50 000 spider bevels. The aggregate number of repetitions $N$ is appreciated 1.2 billion. The computation of the mutual intersection of all spider bevels of the model is happening in every repetition. The computational complexity of the system is at least 60 trillion operations. Based on the threedimensional model of the handling manipulator pattern the model has been ready, description of the frictional contact dynamics of the proximal and distal phalanges of the fingers and from various land areas situated on the plane of the palm.

\subsection{Test items}

In accord with the assignment that have been put before this programme, the next reference items, used for modeling item grasp, were chosen, three-dimensional models of which have been made (Figure 4):

- A cube with aside $30 \mathrm{~mm}$ and aside $60 \mathrm{~mm}$ in length.

- A cylinder with diameter of $30 \mathrm{~mm}$ and of $60 \mathrm{~mm}$.

- A Pencil 7 mm (thick).

- A Ball with a diameter of $30 \mathrm{~mm}$ and of $50 \mathrm{~mm}$.

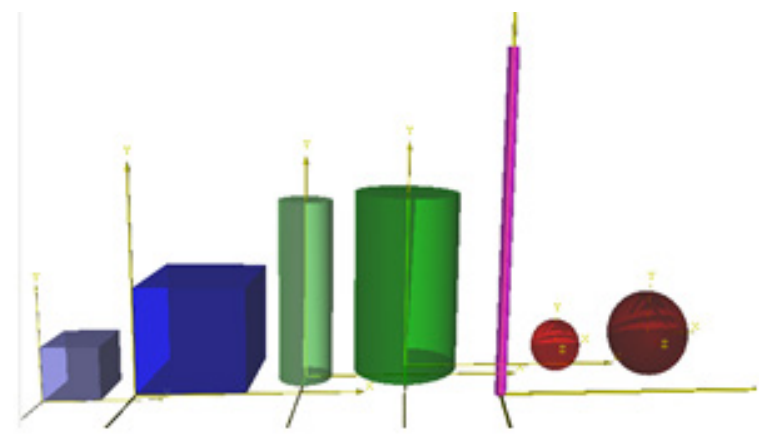

Figure 4. Test objects. These items have been loaded into a GraspIt! environment.

\subsection{Purpose of Experiments}

In this program the simulator GraspIt! ${ }^{17}$ has been used to investigate the kinematics for the advanced manipulator. The package has been used to guide a comparative research of the efficacy of the grip function, for a given set of items by the standard of maximum quality ${ }^{6}$ indices, of the different manipulators.

In the GraspIt! package the following models of resembling a human being manipulators are given:

- Barret Hand.

- Shadow Dexterous Hand.

- Robonaut.

- Meka Hand.

- DLR.

- Shunk hand.
- RobotiQ.

Experimental researches were organized in conformity with the next plan:

1. Introduce the model of the manipulator into the GraspIt! Environment

2. Introduce the item models used to check the grip function.

3. Start the scheduler for gripping with the next indices:

- Energy Formulation = Hand Contacts

- $\quad$ Space Search Type = Axis-angle

- Planner type $=$ Sim. Ann.

- Max Steps $=70000$.

4. (Grasp->Auto Grasp) and (Grasp->Quality Measures...) are used.

It will be observed that during the import of the model of the manipulator and also the object is being grasped, they are placed at the source of the worldwide frame of reference and there is no point to execute any manipulations with virtual models.

During the experimental investigation on the efficacy of functional diagram, the robots models of Robonaut, DLR and a virtual model mimicking the human hand of HumanHand were used.

\section{Results}

As a model of the robot a virtual model of the "RUbionic" human hand with twenty two levels of liberty was used and as an item a model of a sphere with a diameter of fifty mm was used. Total amount of researches (experiments) ten. The calculations for the twenty best gripping variants have been executed from the results of each experiment.

Every manipulator-item pair received a sample of data that consists of a group of twenty of the best variants for gripping models on the standard of quality ratings. The extra-handling phase for the item was executed for each group. At the end of the extra-handling phase the dimension of the last quality rating was done.

\subsection{Statistics and Data Mining}

Results of the conducted research into the induction of the entry conditions of carrying out an experiment are given in Table 1.

Therein after (Table 1),

$A v g=\frac{\sum_{i=1}^{10} \text { quality }_{i}}{10}$ 
Table 1. The result of entry conditions on carrying out the experiment (maximum $=1,00$ )

\begin{tabular}{lcccccccccc}
\hline & $\mathbf{1}$ & $\mathbf{2}$ & $\mathbf{3}$ & $\mathbf{4}$ & $\mathbf{5}$ & $\mathbf{6}$ & $\mathbf{7}$ & $\mathbf{8}$ & $\mathbf{9}$ & $\mathbf{1 0}$ \\
\hline Quality & 0,431 & 0,423 & 0,409 & 0,432 & 0,441 & 0,447 & 0,416 & 0,411 & 0,407 & 0,414 \\
Avg & & & \multicolumn{7}{c}{0,4231} \\
Deviation, \% & 1,87 & 0,02 & 3,33 & 2,10 & 4,23 & 5,65 & 1,68 & 2,86 & 3,81 & 2,15 \\
\hline
\end{tabular}

Table 2. The quality rating of gripping for different manipulators

\begin{tabular}{lccccccc}
\hline Robot model & Cube 30 & Cube 60 & Pencil 7 & Sphere 30 & Sphere 50 & Cylinder 30 & Cylinder 60 \\
RUbionic (human) & 0,0381 & 0,197 & 0,00405 & 0,151 & 0,331 & 0,0371 & 0,241 \\
RUbionic (monkey) & 0,0412 & 0,135 & 0,00314 & 0,127 & 0,342 & 0,0217 & 0,268 \\
DLR & N/A & 0,208 & 0,00308 & N/A & 0,319 & 0,0397 & 0,263 \\
Robonaut & 0,022 & 0,172 & N/A & 0,131 & 0,292 & 0,0411 & 0,0945 \\
Virtual Human hand & 0,0362 & 0,201 & 0,0281 & 0,156 & 0,447 & 0,0471 & 0,203 \\
\hline
\end{tabular}

N/A means that when executing the experiments it was unrealizable to make a successful grip on the item with these measurements.

Deviation $_{i}=\frac{\text { quality }_{i}}{A v g} \cdot 100 \%$

The standard deviation in the deflection of quality ratings doesn't surpass $5.7 \%$. The quality ratings, for which the variety is less than $5.7 \%$, can be considered as equal.

The study about the effectiveness of item gripping from the specified set has been made; the results there are in Table 2.

A lot of experiments with different combinations of manipulators and items were performed (Table 2).

It leads to the conclusion that made kinematic schemes are corresponding to resolve the objective of gripping items from the defined set.

From Table 2 the advanced manipulator copes rationally with the objective of gripping the smaller items and also with grasping the larger items.

The kinematic scheme which uses the monkey scheme has some advantages if we can compare it with for example the human scheme:

- It lets it to grip little items better.

- It makes a better grasp of cylindrical and long items.

Such preferences are the cause of the situation that in most models of motions, when doing manipulations with an item, the principles digits which participate in the grip are the index, the thumb and also the middle finger. Consequently, when the thumb is contrary the index or may be the middle finger, the plane of movement becomes parallel. It makes a more trustworthy grip than in the event that the human scheme. So, it is more effectual to apply the monkey scheme, but if the target audience of items is huge items, so we will apply the human scheme.

\section{Discussion}

As a consequence of a research, the efficacy of the advanced kinematic scheme of the resembling a human being manipulator has been confirmed. Based on the kinematic scheme the three-dimensional model of the manipulator model has been made. During the next phase of this research the pattern manipulator will be done. Following evolution of the manipulation algorithms and their experimental studies will be done using the next approach to the modeling and also straight debugging the pattern. Defined in this study is the methodology of study for manipulator kinematics and it may be used in the starting stage of development for multijointed handling manipulators, also those with other schemes.

The next restrictions have been used during the modeling process for gripping items:

- The kinematics motions of the links of the manipulator have been searched.

- The employment of geometric computations for the collisions of virtual models to define which components come in contact.

The following method with the results of the manipulator modeling will be applied in the following stages of study on the evolution of a resembling a human being manipulator.

As a consequence from the study and research of the received experimental information, and relying on the researches ${ }^{18}$, the evolution of the executive systems will take 
into consideration the situation that the method for gripping items can be shared into different phases (Figure 6):

- Formation of the model for the home position (rough forming).

- Pregrasp (pregrasp).

- Straight grasp (grasp execution).

For gripping items with difficult forms, fragile or supple items, the adaptive synthesis of actions, which comprises the special features of the design and the materials features of the item, can be applied. As a result, for manipulations with items without using information given a priory about the weight, size, texture, the contact dynamics, it demands the building of a difficult system of management and therefore, high requirements in the output of the computational method of the operator. The most significant need to resolve the problem of manipulating a great set of item classifications with certain features becomes the growth of the managing operator. In the next stage of research the group of authors will begin study and research of the manipulation algorithm for the grip and the evolution, development of an adaptive controller.

The implements and modes used in the present phase of the program via the appreciating of the characteristic of the grip given in section 3 will then let to authors to elasticity configure the factors of the kinematic scheme in the design of a worldwide resembling a human being handling manipulator and also manipulators for special requests, for example bionic prosthetics.

\section{Conclusion}

At this stage of the research the method for research and study of the function, operational comfort of the kinematic schemes of the manipulator ${ }^{19}$. Hereafter, to define the optimum number of actuators for the executive links a supplementary research of the impulse on the characteristic of retaining items and kinematic connections between pairs of links will be done and also the importance of the translation ratio between these units.

From the outcomes of this research the evolution of the legacy system for management, path planning of a multi-finger manipulator will be performed. Based on the advanced motion models the embedded legacy system for the executive systems of the anthropomorphic manipulator will be advanced.

\section{Acknowledgments}

This research is financially supported by the Ministry of Education and Science of the Russian Federation under the Grant agreement \# 14.577.21.0136 from 24 November 2014. The research topic: "Development of a universal gripping device for performing anthropomorphic type contact operations with high accuracy and reliability" (Unique reference identifier of the agreement: RFMEFI57714X0136). Work on the project is carried out at the Moscow State University of Mechanical Engineering (MAMI).

\section{References}

1. Roboforce: Chinese plant replaces 90 percent of workers with robots; 2015 May 6. Available from: http://rt.com/ news/256245-china-factory-robot-workforce/ [in English].

2. Chinellato E, Fisher RB, Morales A, Del Pobil AP. Ranking planar grasp configurations for a three-finger hand. IEEE Proceedings of International Conference on Robotics and Automation, ICRA'03; 2003 Sep. p. 1133-8. [in English].

3. Provenzale A, Cordella F, Zollo L, Davalli A, Sacchetti R, Guglielmelli E. A grasp synthesis algorithm based on postural synergies for an anthropomorphic arm-hand robotic system. IEEE International Conference on Biomedical Robotics and Biomechatronics (2014 5th IEEE RAS and EMBS); Sao Paulo. 2014 Aug 12-15. P. 958-63. [in English].

4. Karnati, N, Kent BA, and Engeberg ED. Bioinspired sinusoidal finger joint synergies for a dexterous robotic hand to screw and unscrew objects with different diameters. IEEE/ ASME Transactions on Mechatronics. 2013; 18(2):612-23. [in English].

5. Ciocarlie MT, Clanton ST, Spalding MC, Allen PK. Biomimetic grasp planning for cortical control of a robotic hand. IEEE/RSJ International Conference on Intelligent Robots and Systems, IROS 2008; Nice. 2008 Sep 23-26. p. 2271-6. [in English].

6. Miller AT, Allen PK. Examples of 3D grasp quality computations. IEEE Proceedings International Conference on Robotics and Automation; Detroit, MI. 1999. p. 1240-6. [in English].

7. Lovchik CS, Diftler MA. The robonaut hand: A dexterous robot hand for space. IEEE Proceedings International Conference on Robotics and Automation; Detroit, MI. 1999. p. 907-12. [in English].

8. Grebenstein M, Albu-Schaffer A, Bahls T, Chalon M, Eiberger O, Friedl W, Hirzinger G. The DLR hand arm system. IEEE Proceedings International Conference on Robotics and Automation (ICRA); Shanghai. 2011 May 9-13. p. 3175-82. [in English]

9. Dung LT, Kang HJ, Ro YS. Robot manipulator modeling 
in matlab-simmechanics with PD control and online gravity compensation. IEEE International Forum on Strategic Technology (IFOST); 2010 Oct. p. 446-9. [in English].

10. Crenganis M, Breaz R, Racz G, Bologa O. The inverse kinematics solutions of a 7 DOF robotic arm using Fuzzy Logic. 7th IEEE Conference on Industrial Electronics and Applications (ICIEA); Singapore. 2012 Jan 18-20. p. 518-23. [in English].

11. Dean-Leon E, Nair S, Knoll A. User friendly Matlab-toolbox for symbolic robot dynamic modeling used for control design. IEEE International Conference on Robotics and Biomimetics (ROBIO); 2012 Dec. p. 2181-8. [in English].

12. Tomovic R, Bekey GA, Karplus WJ. A strategy for grasp synthesis with multifingered robot hands. IEEE Proceedings International Conference on Robotics and Automation. 1987 Mar. p. 83-9. [in English].

13. Bernardino A, Henriques M, Hendrich N, Zhang J. Precision grasp synergies for dexterous robotic hands. IEEE International Conference on Robotics and Biomimetics (ROBIO); 2013 Dec. p. 62-7. [in English]
14. Goldfeder C, Ciocarlie M, Dang H, Allen PK. The columbia grasp database. IEEE International Conference on Robotics and Automation ICRA'09; 2009 May. p. 1710-6. [in English].

15. Ciocarlie M, Goldfeder C, Allen P. Dimensionality reduction for hand-independent dexterous robotic grasping. IEEE/RSJ International Conference on Intelligent Robots and Systems IROS 2007; San Diego, CA. 2007 Oct29-Nov 2. p. 3270-5). [in English].

16. Ciocarlie MT, Allen PK. Hand posture subspaces for dexterous robotic grasping. The International Journal of Robotics Research. 2009; 28(7):851-67. [in English].

17. Miller AT, Allen PK. (2004). Graspit! a versatile simulator for robotic grasping. IEEE Robotics and Automation Magazine. 2004; 11(4):110-22. [in English].

18. Wohlke G. Automatic grasp planning for multifingered robot hands. Journal of Intelligent Manufacturing. 1992; 3(5):297-316. [in English].

19. Krechetov I. RUbionic GraspIt! Models; 2015 May 22. Available from: https://yadi.sk/d/S-9axIOugoZ7h [in Russian]. 